\title{
Understanding particle defect transport in an ultra-clean sputter coating process
}

Chris Walton, Patrick Kearney, James Folta, Donald Sweeney, Paul Mirkarimi

U.S. Department of Energy

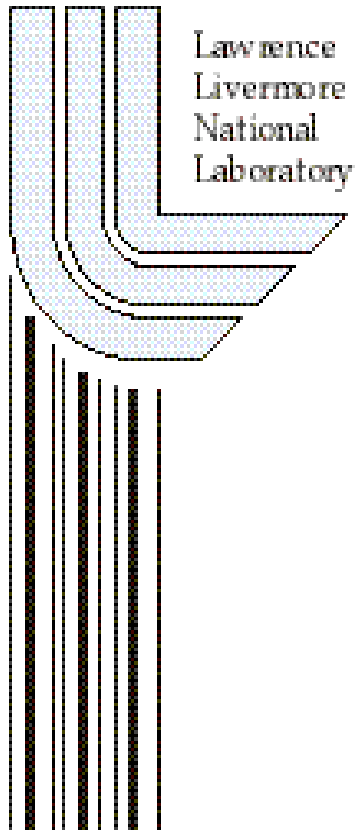

The International Society for Optical Engineering, Santa Clara, February 24, 2003

\section{April 2003}




\section{DISCLAIMER}

This document was prepared as an account of work sponsored by an agency of the United States Government. Neither the United States Government nor the University of California nor any of their employees, makes any warranty, express or implied, or assumes any legal liability or responsibility for the accuracy, completeness, or usefulness of any information, apparatus, product, or process disclosed, or represents that its use would not infringe privately owned rights. Reference herein to any specific commercial product, process, or service by trade name, trademark, manufacturer, or otherwise, does not necessarily constitute or imply its endorsement, recommendation, or favoring by the United States Government or the University of California. The views and opinions of authors expressed herein do not necessarily state or reflect those of the United States Government or the University of California, and shall not be used for advertising or product endorsement purposes.

This is a preprint of a paper intended for publication in a journal or proceedings. Since changes may be made before publication, this preprint is made available with the understanding that it will not be cited or reproduced without the permission of the author.

This report has been reproduced directly from the best available copy.

Available electronically at http://www.doc.gov/bridge

Available for a processing fee to U.S. Department of Energy

And its contractors in paper from

U.S. Department of Energy

Office of Scientific and Technical Information

P.O. Box 62

Oak Ridge, TN 37831-0062

Telephone: (865) 576-8401

Facsimile: (865) 576-5728

E-mail: reports@adonis.osti.gov

Available for the sale to the public from

U.S. Department of Commerce

National Technical Information Service

5285 Port Royal Road

Springfield, VA 22161

Telephone: (800) 553-6847

Facsimile: (703) 605-6900

E-mail: orders@ntis.fedworld.gov

Online ordering: http:/ / www.ntis.gov/ordering.htm

\section{OR}

Lawrence Livermore National Laboratory

Technical Information Department's Digital Library

http:/ / www.llnl.gov/tid/Library.html 


\title{
Understanding particle defect transport in an ultra-clean sputter coating process
}

\author{
Chris Walton*, Patrick Kearney, James Folta, Donald Sweeney, and Paul Mirkarimi \\ Lawrence Livermore National Laboratory, 7000 East Avenue, Livermore, CA 94550 USA
}

\begin{abstract}
Low-defect mask blanks remain a key technical challenge to Extreme Ultraviolet Lithography (EUVL). The mask blank is ion-beam sputter-coated with an 81 -layer Mo/Si multilayer stack for high reflectance at $\square=13.4 \mathrm{~nm}$. The current mask coating process can achieve a median added defect level of 0.05 defects $/ \mathrm{cm}^{2}(12$ added defects $90 \mathrm{~nm}$ or larger on a $200 \mathrm{~mm}$ Si-wafer test substrate), but this must be reduced by about a factor of 10 to meet mask cost requirements for EUVL.

To further reduce the particle defect level, we have studied pathways for particle transport, using test particles and particles native to the coating process, and combined the results into a computational model of particle transport in an ion-beam sputter system. At process pressure, gas drag is negligible for particles above $100 \mathrm{~nm}$, so particles travel ballistically until they hit a surface. Bounce from chamber walls allows particles to reach all surfaces in the chamber if they have initial velocities above $\sim 100 \mathrm{~m} / \mathrm{s}$. The ion beam has sufficient momentum to entrain slower particles and accelerate them toward the sputter target, where some can bounce to the substrate. The model shows preliminary agreement with experimental defect distributions on witness wafers at various positions within the coating chamber.
\end{abstract}

Keywords: EUVL, EUVL mask, defect, multilayer, coating, particle, particle transport, particle adhesion, modeling

\section{BACKGROUND}

Extreme Ultraviolet Lithography (EUVL) is the expected next-generation fabrication method for large-scale IC production at the $30 \mathrm{~nm}$ critical-dimension node and beyond ${ }^{1}$. EUVL is an extension of optical lithography to a wavelength $\square=13.4 \mathrm{~nm}$, in the soft $\mathrm{x}$-ray or extreme ultraviolet regime. Because all solid materials are strongly absorbing at this wavelength, EUVL uses reflective optics rather than lenses. The optics must be coated with multilayer quarter-wave stacks for enhanced reflectivity. The best-performing multilayers to date are 81 -layer $\mathrm{Mo} / \mathrm{Si}$ stacks $(2.8 \mathrm{~nm}$ $\mathrm{Mo}+3.9 \mathrm{~nm}$ Si for a total period thickness of $6.7 \mathrm{~nm}$ ) with normal-incidence reflectivity of $68-70 \%$. The multilayer is typically applied by ion-beam or magnetron sputtering at low pressures (0.1-1mtorr) to achieve a smooth, high density coating of each material.

The EUVL reticle is also a reflective optic, and it is patterned with absorbing material to provide contrast to print IC features. Unlike other optics in an EUVL stepper, however, the mask is on the image plane of the optical system, and defects on the mask can be imaged directly onto the wafer. This places an additional constraint on the process to multilayer-coat a blank reticle: the coating may have at most 1 defect disturbing the multilayer reflectivity, or a defect density of $0.005 / \mathrm{cm}^{2}$ above a critical defect size of about $50 \mathrm{~nm}$.

The Low-Defect Deposition (LDD) tool at LLNL has demonstrated ${ }^{2}$ coating single $200 \mathrm{~mm}$ Si wafers with zero added defects above $90 \mathrm{~nm}$ over a quality area of $10 \square 13 \mathrm{~cm}$, and a series of 20 wafers with a median added particle density of

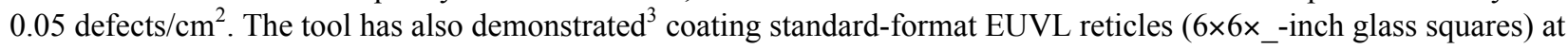
the same particle density as $\mathrm{Si}$ wafers coated in the same lots, indicating the process is transferable from Si to glass substrates at the same defect level. However, a factor of 10 improvement is needed in defect density to reach an acceptable yield and cost for the EUVL reticle. Since the added defects observed are almost exclusively particulates, this paper will consider only particle defects.

\footnotetext{
*Corresponding author: walton9@llnl.gov; phone 1925423 2834, fax 19254231488
} 
Development of the multilayer coating process for the reticle has focused on engineering improvements to the coating chamber, such as an electrostatic substrate chuck and improved finishing processes for the shields and sputter targets. Recently, however, a separate effort has been made to reach more scientific understanding of processes contributing to particle contamination. In addition to processes that produce particles, a transport mechanism is required to deliver the particles to the substrate. Studying the transport mechanisms has proved easier and more conclusive experimentally than studying the sources, and experimental transport studies have given sufficient new knowledge to write a mathematical transport model as well. This paper will describe the experimental studies of particle transport in ion-beam sputter (IBS) systems and the model written to describe it.

\section{EXPERIMENTAL SETUP}

Two ion-beam sputter chambers were used for experiments: the Low-Defect Deposition (LDD) tool used for EUVL mask coating development at LLNL, and a smaller system referred to as the Test Chamber. The LDD tool has been described in detail elsewhere ${ }^{4}$. The Test Chamber is a spherical chamber about $80 \mathrm{~cm}$ in diameter, with 20 observation ports around the surface. The chamber is cryopumped and achieves a base pressure of $5 \square 10^{-7}$ torr. Inside the sphere, about $11 \mathrm{~cm}$ below the equator plane, is a flat stainless-steel platform with an array of threaded holes. All experiments were performed at the level of this platform. Outside the sphere is a $1 \mathrm{~m} \square 2 \mathrm{~m}$ enclosure with HEPA-filtered air flow. The edge of the sphere extends into this enclosure such that the port for loading specimens is in the protected air flow.

The test chamber is also equipped with two seeders for introducing test particles into the sputter process. The first, Particle Source I, is a simple "salt shaker" consisting of a $2 \mathrm{~cm}$-diameter aluminum canister with a finely-perforated metal screen on the bottom. The canister was mounted to a rotatable feed-through which could be vibrated to shake particles through the screen. While this source was easy to construct and operate, it produced clusters of particles rather than single ones, and the particles may have been charged by the vibration.

Particle Source II is a commercial aerosol generation system (TSI Inc., St. Paul, MN) consisting of a liquid reservoir of test particles in DI water, an atomizer (model 3076), two diffusion drying cells, and a charge neutralizer. The water is atomized into droplets, some of which contain a test particle. The droplets pass an impaction plate, which removes the larger droplets, leaving a mean droplet size of $0.3 \square \mathrm{m}$ and at most one particle per droplet. The drying cells then remove moisture by diffusion, leaving only the test particles, which are then reduced to a charge of a few $e$ (positive or negative) by the neutralizer. The particles are then injected directly into the chamber through a $25 \square \mathrm{m}$ orifice in a thin plate (Small Parts, Inc., Miami Lakes, FL) on the end of a flexible copper tube. Because of the large pressure drop through the orifice, the gas flow through it is choked, and the gas in the orifice reaches Mach 1 for argon, or about $320 \mathrm{~m} / \mathrm{s}$. Under these conditions, the particles are accelerated to the speed of the surrounding gas in a short distance (about $500 \square \mathrm{m}$ ). Since this distance is comparable with the thickness of the orifice plate, the particle is expected to reach nearly $320 \mathrm{~m} / \mathrm{s}$ before entering the chamber. This particle source therefore produced individual particles of low charge and known direction and velocity, though the velocity was high.

Epitaxial silicon wafers were used as particle witnesses in the LDD and test chamber. Most wafers were previous specimens from low-defect coating experiments in the LDD tool, and were coated with a standard 81-layer $\mathrm{Mo} / \mathrm{Si}$ multilayer. For sputter targets, uncoated wafers from the same lots were used. All wafers were handled and carried in their original (Integris H9200) boxes and cassettes from the supplier (Wacker Siltronic). The wafers were loaded into the chamber by hand, using clean tweezers and fresh latex gloves as a handling protocol. Using this procedure, wafers could be loaded, pumped, vented, packaged, then unpacked and scanned for particles, with about 10-30 added particles (or about 0.04 to 0.12 per $\mathrm{cm}^{2}$ ) from these processes. The handling and packaging alone (without pumping and venting) added about 0.004 to 0.02 particles $/ \mathrm{cm}^{2}$.

In the Test Chamber, the wafers here held in a vertical position by a single spring clip holding each wafer to a steel post threaded into the chamber floor. In the LDD, wafers were held to the target fixture by similar spring clips, or held in a vertical position on the chamber floor by a free-standing folded sheet-metal stand made of bead-blasted and ultrasonically-cleaned stainless steel. 
The wafers were inspected for particles with a Tencor 6420 optical wafer inspection tool. The sensitivity was about $0.12 \square \mathrm{m}$ PSL equivalent on Si or Mo/Si-coated substrates.

\section{EXPERIMENTS: PARTICLE TRANSPORT DURING COATING}

\subsection{Experiments with witness wafers}

In the first experiments, witness wafers were placed in three key locations in the LDD and test chamber to measure particle deposition. The locations were 1) in the ion beam (i.e. the wafer was used as the sputter target), 2) in the direction of the maximum flux of sputtered material (i.e. as the mask being coated), and 3) in a "hide" position away from the sputter flux and facing away from the target, toward the chamber wall. These are the "Target", "Mask", and "Hide" positions for all experiments described in this paper. The positions are shown in the chamber diagrams in Fig. 1 for two experiments in the LDD ( $a$ and $c$ ) and one in the test chamber $(b)$. Note that in Fig. $1 c$ the hide wafer was not vertical but was facing $45^{\circ}$ down and back, away from the target. In this case the "Mask" and "Hide" wafers were in separate experiments. In all other cases the wafers are vertical and were all exposed simultaneously. The arrows indicate the direction the polished side of the wafer is facing.

The three experiments shown were the following. In experiment 1 (see Fig. 1a), wafers were loaded manually into the LDD tool. The Target wafer was clipped to the target fixture, and the Mask and Hide wafers were held vertically near the chamber floor (therefore about $30 \mathrm{~cm}$ below the plane of the target center) in the positions shown. The chamber was pumped down to its normal base pressure of $\sim 2 \square 10^{-8}$ torr, then the ion beam was run for 3 minutes. The chamber was then vented and the wafers removed. In experiment 2 (Fig. 1b), wafers were hand-loaded into similar positions in the test chamber, then the ion beam was run for 3 minutes, and the wafers removed. In this case the Hide wafer was in front of the Mask wafer (partially obscuring the Mask wafer in order to observe any shadowing effect) but facing the back wall. In experiment 3, wafers were run in the LDD in normal operation, for full multilayer deposition (about 2 hours) under clean conditions. Some were held in the normal coating position facing the target (the Mask position as shown), while for others the chuck fixture was rotated $135^{\circ}$ down and back, leaving the wafer facing away from the target but $45^{\circ}$ down as shown. This was the closest approach to a Hide geometry possible with clean operation of the LDD (i.e. with only robotic wafer loading through the load-lock.)
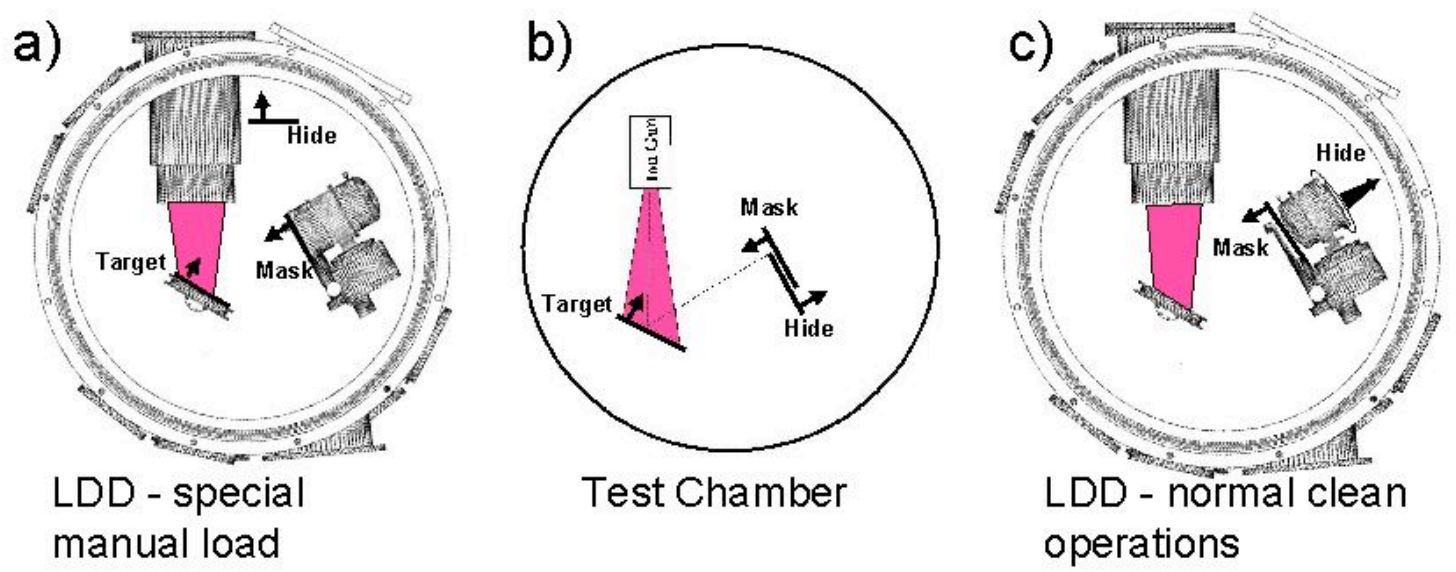

Fig. 1. Top-view diagrams of $a$ ) experiment $1, b$ ) experiment 2, and c) experiment 3. The black bars are 200mm Si wafers used as witness plates, seen edge-on, except in $c$ ), where the Hide wafer is tilted $45^{\circ}$ down and to the right. The wafers in $a$ ) and $b$ ) were all present simultaneously, while the wafers in $c$ ) were placed in the chamber by a handling robot in only one position (Mask or Hide) at a time. No wafers could be placed in the Target position by the robot, so experiment 3 has Mask and Hide wafers only.

Particle counts for the three experiments are shown in Fig. 2. For experiment 1, high particle counts were found on all three wafers. This is consistent with previous results with the LDD tool: after the tool has been vented and opened, 
particle levels are high for the first wafers coated. The highest particle density is found on the Target wafer, followed by

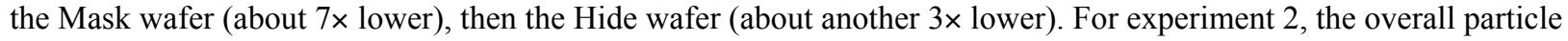
level is lower, possibly because the Test Chamber was open for a much shorter time (about 15 minutes rather than several hours for the LDD), and because a continuous flow of dry $\mathrm{N}_{2}$ gas prevented air from entering the Test Chamber, while the LDD tool was opened from the top and fully exposed to air. However, the particle densities on the three wafers are all reduced by about the same factor (about 100), and the ratios of the particle densities (Target:Mask and Mask:Hide) are approximately the same. Finally, in experiment 3, several wafers were run through the chamber before the experiment; the overall particle level is consequently cleaner by about $500 \square$. For this experiment, the wafers were loaded robotically, so no wafer could be placed on the target, but only one in either the Mask and Hide position. Still, a similar ratio is observed between the Mask and Hide particle densities as in experiments 1 and 2: a ratio of about 5, compared with 2.5-3 in the first two experiments. The similar particle distribution under such different conditions suggests there is a common process distributing the particles in all three cases.

To further investigate this possibility, the particle maps of the wafers from experiment 2 were examined to determine if particles travel by lineof-sight from the target. Line-of-sight transport would be indicated by a strong "shadow" in the Mask particle map, since the Mask wafer is partially obscured by the back of the Hide wafer. The Mask particle map is shown in Fig. $3 c$. The shadow of the circular edge of the Hide wafer is clearly seen, and is indicated by the arrow. However, some particles reached the shadowed region as well, showing not all the particle transport is by line-of-sight. Another remarkable result is the high particle density on the Target wafer in the spot directly hit by the ion beam (see Fig. $3 b$ ). This suggests the ion beam is actively

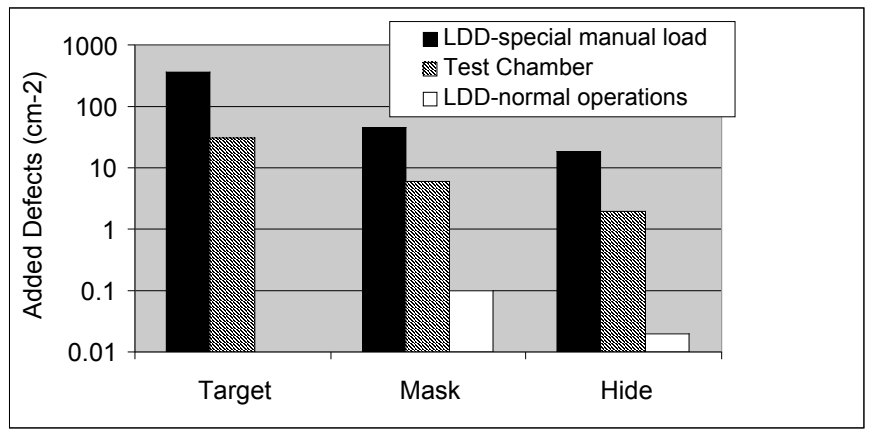

Fig. 2. Added particle counts measured on wafers in the Target, Mask, and Hide positions in the 3 experiments in Fig. 1. Counts include particles above $0.12 \square \mathrm{m}$ PSL equivalent, as measured by optical scatter. All particles are added by the coating process; no particles were seeded in these experiments. transporting or attracting particles to the target. This experiment was repeated 5 times in similar configurations with witness wafers in other places around the chamber, always with the same result: the highest particle density in the beam spot, a lower density on surfaces facing the target, and the lowest density on surfaces facing away from the target. In all cases, however, these low values were at least 2-3 greater than the baseline adder level from pumping and handling, indicating even the low particle levels on the Hide wafers are caused by the sputtering process.

a)

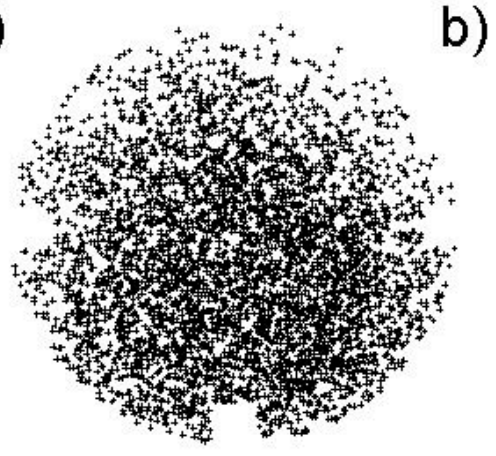

Target b)

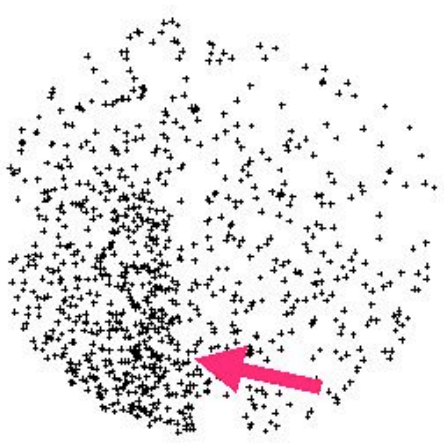

Mask c)

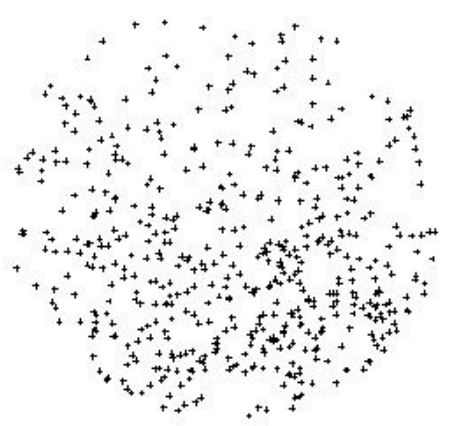

Hide

Fig. 3. Maps of added particles on witness wafers in Experiment 2, on $a$ ) Target, $b$ ) Mask and $c$ ) Hide wafers. These results are for the Test Chamber, under the configuration shown in Fig. $1 b$. 


\subsection{Experiments with "Particle Pinhole Camera"}

In order to establish that these observations were relevant to low-particle coating conditions in the LDD tool, a similar experiment was arranged in that tool. Rather than using a wafer to partially obscure the Mask wafer, a stainless steel shield with a $4 \mathrm{~cm}$ aperture was placed $10 \mathrm{~cm}$ in front of the Mask wafer (see diagram in Fig. $4 a$ ). Any particle travelling by line-of-sight through the aperture would hit the Mask wafer at a spot opposite its point of origin, effectively "imaging" the particle source on the Mask wafer. This "particle pinhole camera" was used in a series of coating experiments. The results for several wafers exposed in the same conditions were superimposed to increase the low signal, since the LDD was operating at a low particle level.

Fig. $4 b$ shows the superimposed results for 5 wafers under normal coating conditions. The area on the Mask wafer with a line-of-sight to the target is indicated by the circle. The particle density there is higher than the background level by $1.5 \square$, where $\square$ is the uncertainty of the particle density in the circular region, calculated as Poisson uncertainty of the integer particle count. No other regions of the chamber "visible" to the Mask wafer show an elevated particle level. On this indication that particles were coming from the target direction, the target was rotated so the ion beam struck it at $70^{\circ}$ from the normal, rather than the usual $30^{\circ}$ angle, on the hypothesis that the beam might produce more particles if it struck a fresh area around the edges of the target. This produced the results in Fig. $4 c$ (again the map is that of 5 wafers superimposed). There is now a strong spot corresponding to the target, standing out from the background by almost 3 standard deviations.
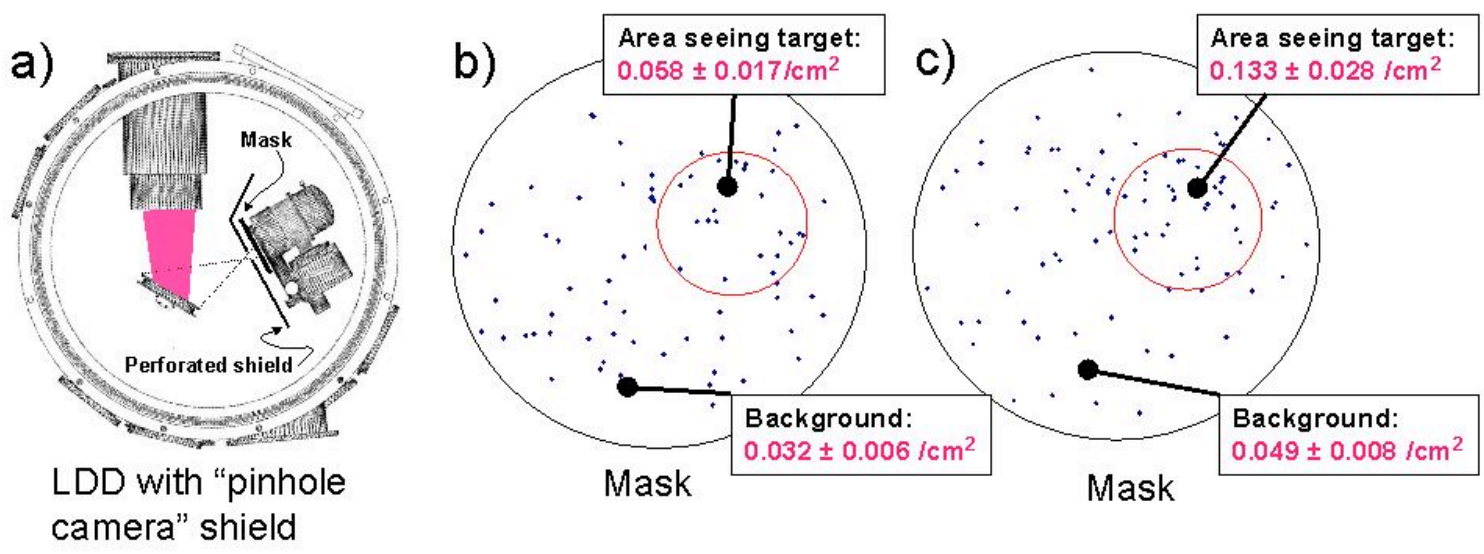

Fig. 4. a) Configuration (top view) of "particle pinhole camera" experiment in Low Defect Deposition (LDD) tool. b) The resulting particle map of 5 Mask wafers combined, for the normal target angle shown in a) (beam hits target at $30^{\circ}$ from target normal). There is a statistically increased particle level in the spot on the wafer with a line of sight to the target, indicated by the circle. $c$ ) repeat of $b$ ) with the target rotated clockwise so beam impacts it at $70^{\circ}$ from the target normal. In this geometry part of the beam strikes the edge of the target, which may account for the increased level of particles arriving from the target onto the wafer.

From the final particle distributions in both coating chambers three facts stand out. First, the spot on the target struck by the ion beam has the highest particle density anywhere in the chamber, suggesting the beam is transporting particles there. Second, particles also leave from the target area and move toward the mask, as shown by the shadowing or line-ofsight effects seen in both chambers. Third, some particle are not limited by line-of-sight travel, as shown by the particle density on surfaces hidden from the target. Based on these results, we investigated whether the ion beam can transport particles to the target, and whether particles impacting the target could bounce and land elsewhere in the chamber. Two supporting experiments to investigate these phenomena are described in the next section. 


\section{SUPPORTING EXPERIMENTS: ENTRAINMENT AND BOUNCE}

\subsection{Deflection of test particles by the ion beam}

To investigate entrainment of particles in the ion beam, a simple experiment was done to sprinkle test particles into the beam and measure their final position. Using Particle Source I containing 1.5 $\square \mathrm{m} \mathrm{SiO} 2$ spheres (Duke Scientific, Palo Alto, CA), particles were dispensed from $8 \mathrm{~cm}$ above the beam (in the Test Chamber), with the beam parallel to the chamber floor as shown in Fig. $5 a$. Eleven centimeters below the beam was a strip of black paper. After the experiment, the paper was removed from the chamber, and the size of the particle clusters measured as a function of position, using optical micrographs of various locations on the paper. It was found there was a smooth dependence: the largest particles were only slightly deflected by the ion beam, showing a small deflection angle $\square$, and the smallest particles had been deflected the most. The data for deflection angle vs. cluster size are shown in Fig. $5 b$ (red points).
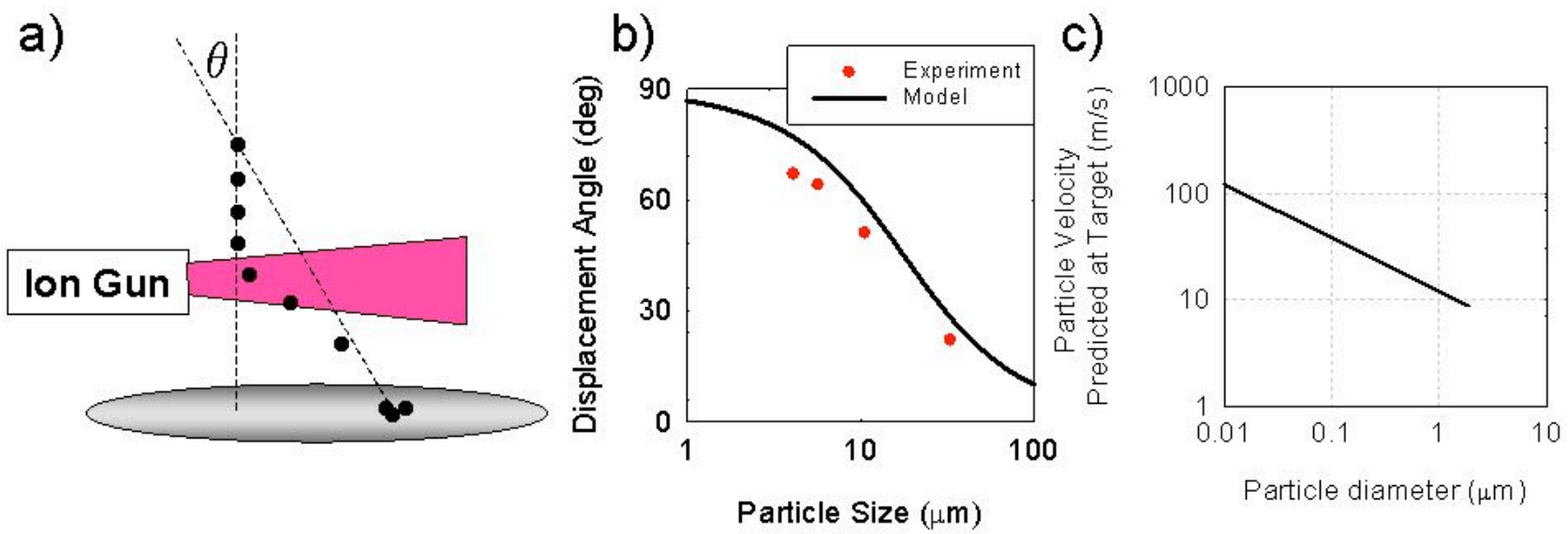

Fig. 5. a) Configuration (side view) of particle deflection experiment in Test Chamber. Particles (1.5 $\square \mathrm{m} \mathrm{SiO}_{2}$ spheres) were dispensed in clusters into the ion beam and their deflection angle $\square$ measured by collecting them below. $b$ ) Deflection angle $\square$ vs. the particle cluster size as measured (points) and calculated with Equation 1 (smooth curve). c) Expected velocity of a particle entering the top of the beam near the ion gun grid, when it reaches the target $38 \mathrm{~cm}$ away, as a function of particle size. The curve ends near particle size $=2 \square \mathrm{m}$; particles larger than this fall through the beam and do not reach the target. Particle density assumed to be $2.2 \mathrm{~g} / \mathrm{cm}^{3}(e . g$. Si or $\left.\mathrm{SiO}_{2}\right)$

The acceleration of micron-sized particles by energetic ions has been observed by other investigators in the case of ion implantation ${ }^{5}$. In this case an approximate explanation could be made with a simple model based on the momentum transferred by the energetic ions in the beam to the particle. If we make the simplest assumption that each ion's momentum is completely transferred to the particle (i.e. the collision is totally inelastic), the momentum change of the particle from each ion impact is $\square p=\sqrt{2 m_{A r} E_{A r}}$, where $\mathrm{m}_{\mathrm{Ar}}$ is the mass of the ion, and $\mathrm{E}_{\mathrm{Ar}}$ its kinetic energy. If the ion beam has a current density $\mathrm{J}\left(\mathrm{m}^{-2} \mathrm{~s}^{-1}\right)$ and the collision cross-section is assumed to be the physical cross section of the particle, then the particle is struck each second by $\mathrm{N}=\square \mathrm{r}_{\mathrm{p}}{ }^{2} \mathrm{~J}$ ions, where $\mathrm{r}_{\mathrm{p}}$ is the particle radius. The force on the particle is then

$\mathrm{F} \equiv \mathrm{d} p / \mathrm{dt}=\mathrm{N} \square p=\square r_{p}^{2} J \sqrt{2 m_{A r} E_{A r}}$.

Using this force during the time the particle is in the ion beam, and writing the equation of motion including gravity, the deflection angle could be calculated as a function of particle size. The result is shown in Fig. $5 \mathrm{~b}$ as the solid curve. The model explains the results to within about a multiplicative factor of 2 in the particle size. This error could result from a systematic error in measuring the average particle size from the micrographs, some tendency of the particles to break up 
in the beam, some of the ions' kinetic energy being transformed into heat rather than kinetic energy of the particle, or a deviation of the actual scatter cross-section from the physical cross-section. There is also some divergence of the ion beam in the Test Chamber, because the grids are flat and not aligned for beam focusing. This would reduce the current density and cause the model to predict too high a deflection angle, as was observed. Divergence of the ion beam and dissipation of some of the ions' energy as heat were addressed in the development of a more complete particle transport model, which is discussed in Section 6.

\subsection{Multiple bounce of high-velocity test particles}

To investigate particle bounce, a simple experiment was performed with the aerosol particle seeder, Particle Source II. Experiments described above in the LDD and the Test Chamber have shown that many particles originate from the direction of the target, but that substantial numbers of particles arrive at surfaces with no line-of-sight to the target. This suggests that some of the particles make a first impact but do not stick, and make at least 1 more trajectory before sticking to another surface. To test this hypothesis in the geometry of a sputtering chamber, two wafers were exposed to $0.5 \square \mathrm{m}$ polystyrene latex (PSL) spheres emitted by Particle Source II as shown in Fig. 6. One wafer was directly in front of the jet of test particles; the second was in a Hide position. At a chamber pressure typical of ion-beam sputtering ( $5 \square$ $10^{-4}$ torr) the results were surprising - rather than a spot of heavy particle deposition in the center of the main wafer, both wafers had a uniform coating of test particles, and the particle count was about the same on both (Fig. 6b).
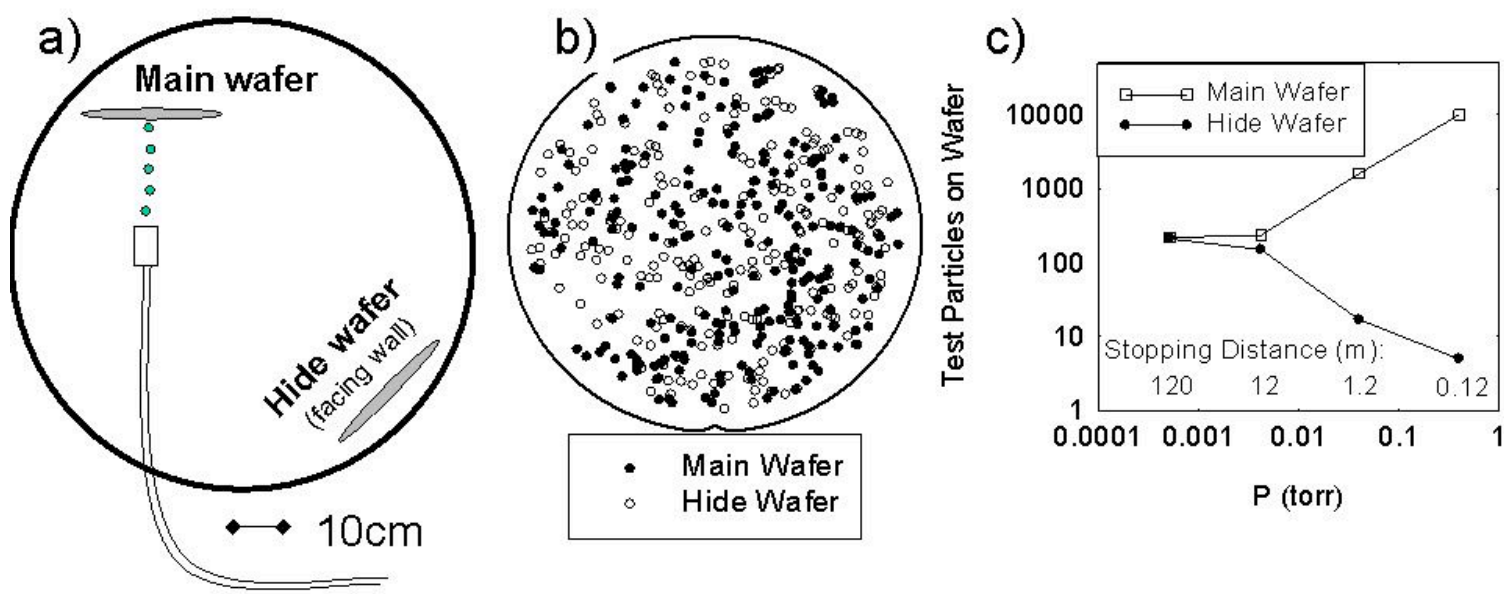

Fig. 6. a) Configuration (top view) of particle bounce experiment in Test Chamber. Latex (PSL) spheres of 0.5 $\square \mathrm{m}$ diameter were injected into the chamber at $\mathrm{P}=5 \square 10^{-4}$ torr toward the main wafer. $b$ ) Resulting particle distributions on the Main and Hide wafers (superimposed on a single map). The particle counts on both are approximately equal and uniformly distributed. $c$ ) Effect of increased pressure on particle counts.

Next the experiment was repeated at higher pressures, where the background gas in the chamber would be expected to slow the particles and prevent a long, bouncing path to a remote chamber surface. As the pressure increased, the particle count on the two wafers diverged. At $\mathrm{P}=5 \square 10^{-1}$ torr, where the viscous drag would stop a $300 \mathrm{~m} / \mathrm{s}$ particle in about $12 \mathrm{~cm}$ (comparable to the distance from the particle jet to the Main wafer), the density of test particles on the Main wafer increased by about 45马, and that on the Hide wafer decreased to a low background level of a few particles (Fig. 6c). A reasonable explanation is the following: the test particles, travelling at $\mathrm{V} \sim 300 \mathrm{~m} / \mathrm{s}$, strike the Main wafer, but are moving too fast to stick, and instead make several bounces after the first impact. At $\mathrm{P}=5 \square 10^{-4}$ torr, the background gas provides no resistance, and the particles end up randomly distributed over the chamber walls and the two wafers. As the pressure is increased, the particles can cover less distance before slowing down and sticking, so fewer make enough bounces to reach the Hide wafer. Finally, at $\mathrm{P}=5 \square 10^{-1}$ torr, most particles are nearly stopped by the time they hit the Main wafer, and they stick there. The particles are now confined to the Main wafer, or about $60 \square$ less surface area than the total inside area of the chamber, explaining why the particle count is about $45 \square$ higher. The discrepancy in the ratios may indicate that even at this pressure not all particles stick to the Main wafer. 


\subsection{Discussion - particle bounce}

Particle bounce has been observed by many investigators ${ }^{6-12}$, with some of the pioneering early work done by Dahneke ${ }^{13}$. The simple case of spheres impacting a smooth flat surface has received the most investigation both experimentally and theoretically. A typical result for this case is shown in Fig. 7a (solid curve), showing the fraction of velocity retained by a 1.3ロm PSL sphere after bounce from a smooth quartz substrate, measured by Dahneke using laser Doppler velocimetry (LDV). At the highest velocities, plastic deformation dissipates a large part of the particle's kinetic energy, so the velocity retained is low: a $300 \mathrm{~m} / \mathrm{s}$ sphere will be slowed to $60 \mathrm{~m} / \mathrm{s}$. Slower particles at V $\sim 20 \mathrm{~m} / \mathrm{s}$ keep nearly all their velocity. For all impacts, energy is lost to adhesion from the surface energy of the particle and surface in the contact area. At the lowest velocities, this becomes a significant fraction of the incoming kinetic energy, and the velocity retained drops. With decreasing velocity, eventually adhesion dominates and the particle sticks for velocities below a critical value, about $2 \mathrm{~m} / \mathrm{s}$ for this case. Theoretical models of this process have been published by several authors ${ }^{6}, 10,14-$ ${ }^{17}$, though there is no definitive theory on the subject. Note that a $300 \mathrm{~m} / \mathrm{s}$ latex sphere in this experiment would be at $\mathrm{V}=$ $60 \mathrm{~m} / \mathrm{s}$ after the first bounce, $50 \mathrm{~m} / \mathrm{s}$ after the second, $43 \mathrm{~m} / \mathrm{s}$ after the third, and would make about 45 bounces before reaching the critical velocity near $2 \mathrm{~m} / \mathrm{s}$.
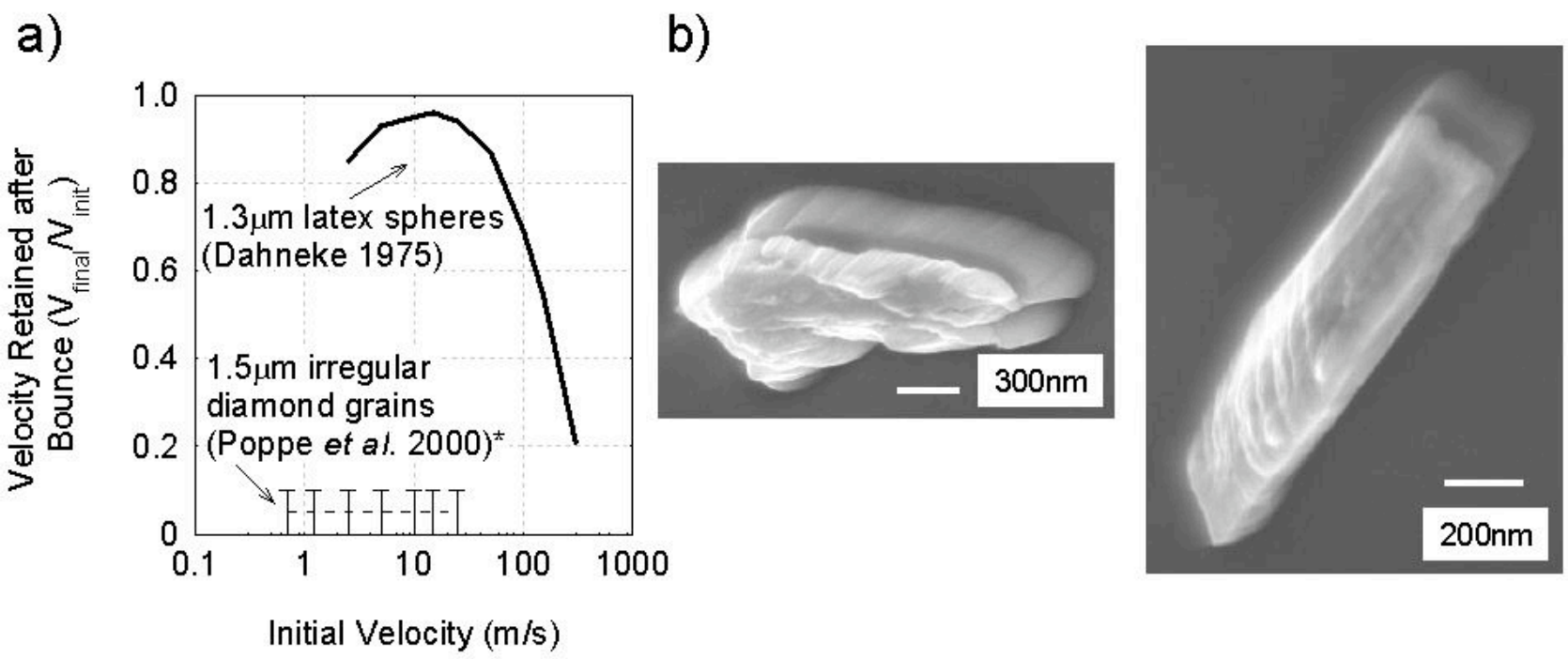

Fig. 7. a) Velocity retention after bounce for spheres (Results by Dahneke ${ }^{13}$, solid curve) and non-spherical grains (Poppe et al. ${ }^{8}$, dashed line) for 1.5 m particles hitting a smooth quartz surface. *Results by Poppe et al. determined only that $\mathrm{V}_{\text {final }} / \mathrm{V}_{\text {init }}<0.1$. This is indicated with light curve and error bars. b) SEM micrographs of particles from Experiment 2 in this paper, captured on the Target wafer in Fig. $1 b$, showing that particles in the sputtering chambers are non-spherical.

For non-spherical particles, much less is known experimentally. An important study by Poppe et al. ${ }^{8}$ measured impact of a variety of irregular grains of minerals, as well as $\mathrm{SiO}_{2}$ spheres, on quartz and silicon surfaces using a strobe photography technique. The study found a dramatic difference between spheres and non-spheres in both the probability of sticking and the velocity retained after bounce. The velocity retained was in most cases too low to be measured accurately, which meant it was below 0.1 for most impacts. This was true over a range of grain sizes from $0.14 \square \mathrm{m}$ to $1.5 \square \mathrm{m}$, in contrast to the high fraction of velocity retained by Dahneke's $1.3 \square \mathrm{m}$ particles in the solid curve. This result is represented in Fig. 7a by the dotted curve, covering the range [0-0.1] for the velocity retained. For non-spheres, the dependence of sticking probability on velocity was much less pronounced. There was no clear cutoff between sticking and bouncing, though the sticking probability generally reached $80 \%$ for velocities of about $1 \mathrm{~m} / \mathrm{s}$. The authors attributed this to the likelihood of multiple contact points when an irregular grain hits a flat surface, and to the possible transfer of some of the particle's translational kinetic energy into rotational energy. 
All of the particles observed on EUVL mask blanks have been non-spherical, with an average aspect ratio (as measured from SEM images) of 1.8. If this is also true of typical particles moving in the chamber during coating, a large velocity loss at each impact, like that seen by Poppe et al., would be expected. Fig. $7 b$ and $c$ show micrographs of two typical particles captured on the target in experiment 2 in the Test Chamber. They are clearly non-spherical, and resemble the flake-shaped particles found on mask blanks coated in the LDD. If they experience a velocity loss of $90 \%$ with each bounce like the grains observed by Poppe et al., even a $300 \mathrm{~m} / \mathrm{s}$ particle needs only $2-3$ bounces to reach $\mathrm{V}=1 \mathrm{~m} / \mathrm{s}$, where sticking is very likely. This can explain a much lower particle concentration on hidden surfaces that are only accessible after at least 1 bounce off the walls, if the particles originate near the target.

\section{POSTULATED PARTICLE TRANSPORT PATH}

Based on the results above, a transport path for particles in ion-beam deposition chambers can be constructed. Particles in the $0.1 \square \mathrm{m}$ to $10 \square \mathrm{m}$ size range can travel freely at $\mathrm{P}=1 \square 10^{-4}$ torr without impedance from drag from the background gas. A particle entering the ion beam will be strongly accelerated by it. Based on the simple equation given above for the ion beam force, a particle accelerated through the full length of the beam can reach a velocity of $10-50 \mathrm{~m} / \mathrm{s}$ at the sputter target (Fig. 5c), though any particles spending less time in the beam would be accelerated less. But from established results on particle bounce, a non-spherical particle at this speed is likely to bounce off the target, and possibly make 1 or 2 further bounces. This would result in a "spray" of particles apparently emanating from the target, with many sticking to the next surface they hit. A smaller number of particles would bounce further, and could reach remote surfaces such as the Hide wafers. This hypothesis is consistent with all the evidence presented: the high density of particle in the ionbeam footprint, a lower density on surfaces facing the target, such as the mask, an apparent arrival direction from the target, and some particles bouncing to remote surfaces. A computational model was constructed to test this hypothesis, and is outlined in the next section.

\section{COMPUTATIONAL MODEL FOR PARTICLE TRANSPORT}

A quantitative model was needed to test whether ballistic particle motion, particle bounce from chamber fixtures, and ion-beam entrainment could explain the particle distributions observed on the various witness wafers placed in the chamber. This section will describe the functioning of this model.

The model assumes an initial size and position of a particle, calculates its trajectory (influenced by ion beam acceleration, gravity, and bounce) until it sticks to a surface, then performs a Monte Carlo repetition for a large number of particles until a final distribution of particles around the chamber is predicted. Since the actual source of particles in these chambers is not known, various scenarios have to be tested, and the predictions compared with experiment. The initial size and velocity are assumed as part of a given scenario under test, and the initial size distribution is assumed to that observed on actual mask blanks coated in the LDD.

The ion beam is modeled by Equation 1 above. The beam is assumed to be Gaussian in profile, and its width changes along the length of the beam as we have measured in simple erosion experiments in the Test Chamber and the LDD.

Particle bounce is modeled according to the results by Poppe et al. A fit was made of those results for the sticking probability of non-spheres as a function of particle size and velocity. The fraction of velocity retained is assumed to be 0.08 , taken from their observation that this quantity is less than 0.1 for irregularly-shaped particles. The model chooses random numbers to decide if bounce occurs. If the particle bounces, a random new direction is chosen for bounce from a rough surface (e.g. the chamber walls), and a near-specular direction is chosen for bounce from a smooth surface (e.g. the target or mask).

Particle motion is iterated along fixed distance steps (typically $2 \mathrm{~mm}$ ), with the time steps chosen accordingly. At each distance step the total force on the particle is calculated (from the ion beam plus gravity), the acceleration vector is calculated from the force and particle mass, the velocity is updated, then the particle is propagated to the next point. A check is made whether it has hit any surface. If impact has occurred, the bounce direction and updated velocity are calculated as described above, then the particle is propagated again. 
The effect of the impact angle on the velocity retained has not been measured for non-spherical particles. For the purposes of the model, the dependence is assumed to be the same as for spheres, which has been measured by Brach et $a l .6$

As an initial working assumption, the model neglects electrostatic forces on the particle. While this point requires further investigation, a negligible electrostatic force is plausible if the electric fields in the chamber and the average charge on the particles are small. The overall ion beam (though not the individual ions) is expected to be nearly charge neutral in both chambers, because of the grounded target and the surrounding metal fixtures providing a large supply of secondary electrons. This neutralization reduces any field along the length of the beam. Particle charging is expected to be maximal in the beam, where the electron density is highest, but any particles charged there would be neutralized again on striking the grounded target. Models in the literature of particle charging in sputter plasmas ${ }^{18,}{ }^{19}$ may be applicable to ion-beam sputtering conditions, but this requires detailed knowledge of the ion and electron densities in the plasma and the local electric field in the chamber. These quantities have not been measured in the LDD plasma, so as a first attempt this is beyond the scope of our work.

\section{PRELIMINARY MODEL RESULTS}

As a first test, the model was applied to the Test Chamber experiment, to determine if any plausible starting scenario (initial particle positions and velocities) matched the observed particle distribution on the three wafers. The configuration modeled was that shown in Fig. $1 b$, except that the shadowing of the Mask wafer by the Hide wafer was not simulated: the Hide wafer was taken to be the backside of the Mask wafer. Several scenarios were modeled, including particles coming from the ion gun, from the target, from the chamber ceiling, and nucleating in the plasma. The best match among these preliminary scenarios was for particles originating from the target with a velocity of about $300 \mathrm{~m} / \mathrm{s}$. Such a velocity is realistic if a stressed film of redeposited material is present around the edges of the target, and such redeposited material is observed on the Si target during regular operation. The predicted wafer maps for the Target, Mask and Hide in this scenario are shown in Fig. 8b, alongside the corresponding experimental results. Note that the calculation time for the simulation was not scaled to match the total number of particles observed in the experiment, so the particle densities differ from experiment by a multiplicative constant.

The results reproduce the experiment in several aspects. The particle density is highest on the target and drops from target to mask to hide. The experimental and theoretical particle densities are compared directly in the bar chart in Fig. $8 a$. The simulation also reproduces the spot of high particle density in the beam footprint on the target. Finally, the experiment shows a higher particle density at the bottom of the Mask wafer than at the top, by a factor of 2-3, and the model reproduces this.

It should be emphasized that this is a preliminary result of the modeling effort, and we cannot conclude yet that most particles in the LDD coating process originate at the target at $\mathrm{V} \sim 300 \mathrm{~m} / \mathrm{s}$ as the model scenario assumed. Further validation of the model against a larger set of experimental results is needed, and the uniqueness of the match between model prediction and experiment must be investigated further. However, the results do establish that there is a selfconsistent approximate explanation of the particle distributions that have been repeatedly observed in both the Test Chamber and the LDD. This explanation rests on the phenomena of ion-to-particle momentum transfer and particle bounce, both of which are known from work by other investigators, and have been verified in the context of ion-beam sputtering in this work.

\section{CONCLUSIONS}

The ion beam in ion-beam sputter (IBS) chambers can deflect or entrain particles in the 3-30 $\square \mathrm{m}$ size range along the direction of ion flux, and the smallest particles are deflected most. A simple model based on momentum transfer from the ions to the particles can account for the results and predicts smaller particles will be fully entrained in the beam, and accelerated to velocities of tens of meters/sec in the typical length of the beam in a sputtering chamber.

A consistent pattern of the distribution of particle contamination has been observed in two different IBS chambers operating at a range of contamination levels. Particles following this distribution travel predominantly by line-of-sight 
paths coming from the area of the sputter target, though a substantial fraction follow non line-of-sight paths to remote areas of the chamber. The non line-of-sight transport is consistent with the known tendency of submicron particles to bounce at velocities above a few meters/sec.

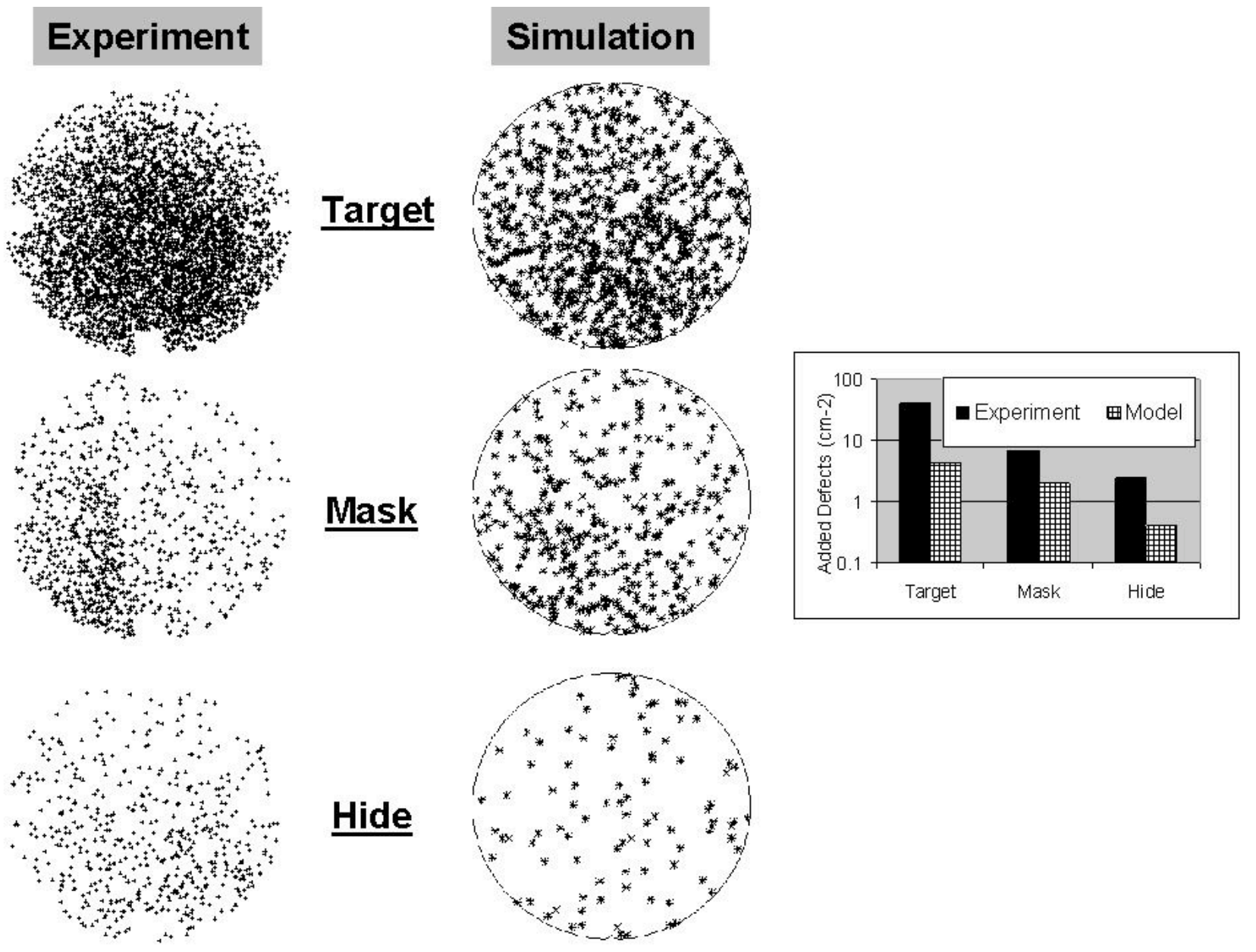

Fig. 8. Direct comparison of experimental and simulated particle counts on Target, Mask and Hide wafers in Experiment 2 (experimental configuration from Fig. $1 b$ ). The simulation reproduces the decreasing particle density from Target to Mask to Hide, though ratios are not exact. There is a higher particle density at the bottom of the Mask wafer that at the top, which is also reproduced by the simulation. The shadowing effect of the Hide obscuring part of the Mask is not expected on simulation, as described in the text. Simulation not scaled to experiment in overall particle count.

The consistent particle distribution suggests there is a common transport mechanism that at least plays an important role in determining the particle distribution in both chambers, even though the particle sources are unknown. In this mechanism, transport is dominated by the ion beam gathering and accelerating particles toward the target, and particle bounce dispersing them. The ion beam entrainment can be explained by momentum transfer from the ions to the particles, though electrostatics may play a role that has not yet been quantified. A particle transport model incorporating these effects approximately explains final particle distributions on 3 witness wafers around the ion-beam sputter chamber, including the relative particle densities from wafer to wafer and the spatial distribution on the wafers. If the model can be validated with additional experiments, it is expected to give new understanding of how a cleaner ion-beam sputter process can be designed. 


\section{ACKNOWLEDGEMENTS}

The authors would like to thank Daniel Stearns for valuable criticism and support in formulating the transport model.

Work performed under the auspices of the U. S. Department of Energy by the Lawrence Livermore National Laboratory under Contract No. W-7405-ENG-48. Funding was provided by the Extreme Ultraviolet Limited Liability Corporation under a Cooperative Research and Development Agreement. Partial funding for the simulation was provided by International Sematech under contract \# LITH-143.

\section{REFERENCES}

1. J. Canning, in: Attendee Survey, Next Generation Lithography Workshop, International Sematech, August 2830, 2001, Pasadena, CA pp. 6-10 at: http://www.sematech.org/public/resources/litho/ngl/ng10901/2001\%20WS\%20Final\%20Report\%202.pdf (2001)

2. J. A. Folta, J. Davidson, C. C. Larson, C. C. Walton and P. A. Kearney, Proc. SPIE 4688, 173-181 (2002)

3. J. A. Folta, P. A. Kearney, C. C. Larson, M. K. Crosley, E. Fisch and K. Racette, Proc. SPIE 4889, 374-381 (2002)

4. $\quad$ P. A. Kearney, C. E. Moore, S. I. Tan, S. P. Vernon and R. A. Levesque, 15, 2452-2454 (1997)

5. $\quad$ D. A. Brown, P. Sferlazzo, S. E. Beck and J. F. O'Hanlon, J. Appl. Phys. 71, 2937-2944 (1992)

6. R. M. Brach, P. F. Dunn and X. Y. Li, J. Adhesion 74, 227-282 (2000)

7. $\quad$ Y. S. Cheng and H. C. Yeh, Environ. Sci. Technol. 13, 1392-1396 (1979)

8. T. Poppe, J. Blum and T. Henning, Astrophys. J. 533, 454-471 (2000)

9. $\quad$ K. T. Paw U, 93, 442-452 (1983)

10. L. N. Rogers and J. Reed, J. Phys. D, Appl. Phys. 17, 677-689 (1984)

11. M. Xu and K. Willeke, J. Aerosol. Sci. 24, 19-30 (1993)

12. S. Wall, W. John, H. C. Wang and S. L. Goren, Aerosol Sci. Technol. 12, 926-946 (1990)

13. B. Dahneke, J. Colloid Interface Sci. 51, 58-65 (1975)

14. K. L. Johnson, K. Kendall and A. D. Roberts, Proc. R. Soc. Lond. A, Math. Phys. Sci. (UK) vol.324, 301-313 (1971)

15. B. V. Derjaguin, V. M. Muller and P. Toporov Yu, $\underline{53}$, 314-326 (1975)

16. B. Dahneke, Aerosol Sci. Technol. 23, 25-39 (1995)

17. D. Maugis, J. Colloid Interface Sci. 150, 243-269 (1992)

18. D. A. Brown, P. Sferlazzo and J. F. O'Hanlon, J. Vac. Sci. Technol. A-Vac. Surf. Films 9, 2808-2812 (1991)

19. J. E. Daugherty, R. K. Porteous and D. B. Graves, J. Appl. Phys. 73, 1617-1620 (1993) 
University of California

Lawrence Livermore National Laboratory

Technical Information Department

Livermore, CA 94551

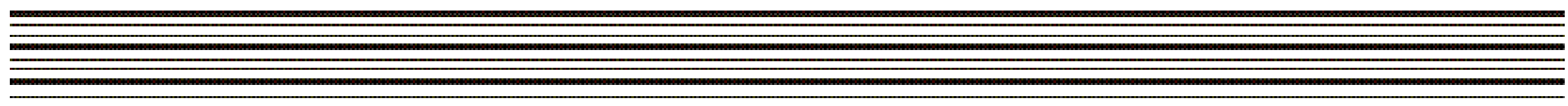

